Radiologe 2022 $62: 109-119$

https://doi.org/10.1007/s00117-021-00953-w

Angenommen: 8. Dezember 2021

Online publiziert: 12. Januar 2022

○ Der/die Autor(en) 2022

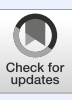

\title{
Pulmonale Rundherde und Pneumonie
}

\author{
Ein diagnostischer Leitfaden
}

Thomas Frauenfelder · Anna Landsmann

Institut für Diagnostische und Interventionelle Radiologie, Universitätsspital Zürich, Zürich, Schweiz

Zusammenfassung

Hintergrund: Das konventionelle Röntgenbild zählt zu den am häufigsten durchgeführten radiologischen Untersuchungen. Seine Interpretation gehört zu den Grundkenntnissen jedes Radiologen.

Fragestellung: Ziel dieses Artikels ist es, häufige Zeichen und Muster der Pneumonie sowie Merkmale von Pseudoläsionen im konventionellen Röntgenbild zu erkennen und einen diagnostischen Leitfaden für junge Radiologen zu schaffen.

Methoden: Analyse aktueller Studien und Daten sowie eine Übersicht der häufigsten Zeichen und Muster im konventionellen Röntgenbild.

Ergebnisse: Die Kenntnis über häufige Zeichen und Muster im Röntgenbild bietet eine Hilfestellung in der Diagnostik und kann hinweisend für die Ursache einer Infektion sein. Häufig sind diese Zeichen jedoch unspezifisch und sollten daher immer in klinische Korrelation gesetzt werden. In der Detektion und Beurteilung von pulmonalen Rundherden gewinnt die Computertomographie (CT) durch ihre deutlich höhere Sensitivität in der Primärdiagnostik immer mehr an Bedeutung.

Schlussfolgerung: Das konventionelle Röntgenbild bildet weiterhin eine führende Rolle in der Primärdiagnostik; der Radiologe sollte jedoch die Limitationen des konventionellen Bildes kennen.

\section{Schlüsselwörter}

Lunge · Infektion · Computertomographie · Röntgenbildgebung · Thorax

Seit Entdeckung der Röntgenstrahlung im Jahr 1895 zählt das konventionelle Röntgenbild zu den häufigsten durchgeführten radiologischen Untersuchungen. Günstig und schnell verfügbar, verschafft es dem Radiologen eine schnelle Übersicht und ist daher unverzichtbar. Mit etwa 9\% der Untersuchungen, steht die Röntgen-ThoraxAufnahme an Platz 3 der jährlich durchgeführten Röntgenuntersuchungen, trägt jedoch durch ihre vergleichbar geringe Strahlendosis nur zu 1\% der jährlichen Strahlenbelastung in der Bevölkerung bei [1].

Nichtsdestotrotz ist das Röntgenbild in seiner diagnostischen Sensitivität deutlich eingeschränkt und variiert, je nach Quelle, sehr stark. Während für die Pneumonie eine Sensitivität von 32-78\% berichtet wird, liegt die Spezifität bei 60-94\% [2, 3]. Beim Nachweis pulmonaler Läsionen liegt die angegebene Sensitivität mit 18-46\% wesentlich darunter und zeigt eine deutliche Abhängigkeit von der Erfahrung des Untersuchers, weshalb sie für ScreeningUntersuchungen ungeeignet ist [4, 5].

Seine Interpretation, aber auch das Wissen über die Limitationen des Röntgenbildes gehören daher zum Standardwissen jedes Radiologen. Ziel dieses Artikels ist es, sowohl charakteristische Zeichen und Muster für Pneumonien und Pseudoläsionen im Röntgenbild zu erkennen als auch einen diagnostischen Leitfaden für junge Radiologen zu schaffen.

\section{Pneumonien - die häufigsten Zeichen}

Die Pneumonie ist eine der häufigsten erworbenen Infektionen im ambulanten Umfeld und stellt die dritthäufigste Ur- 


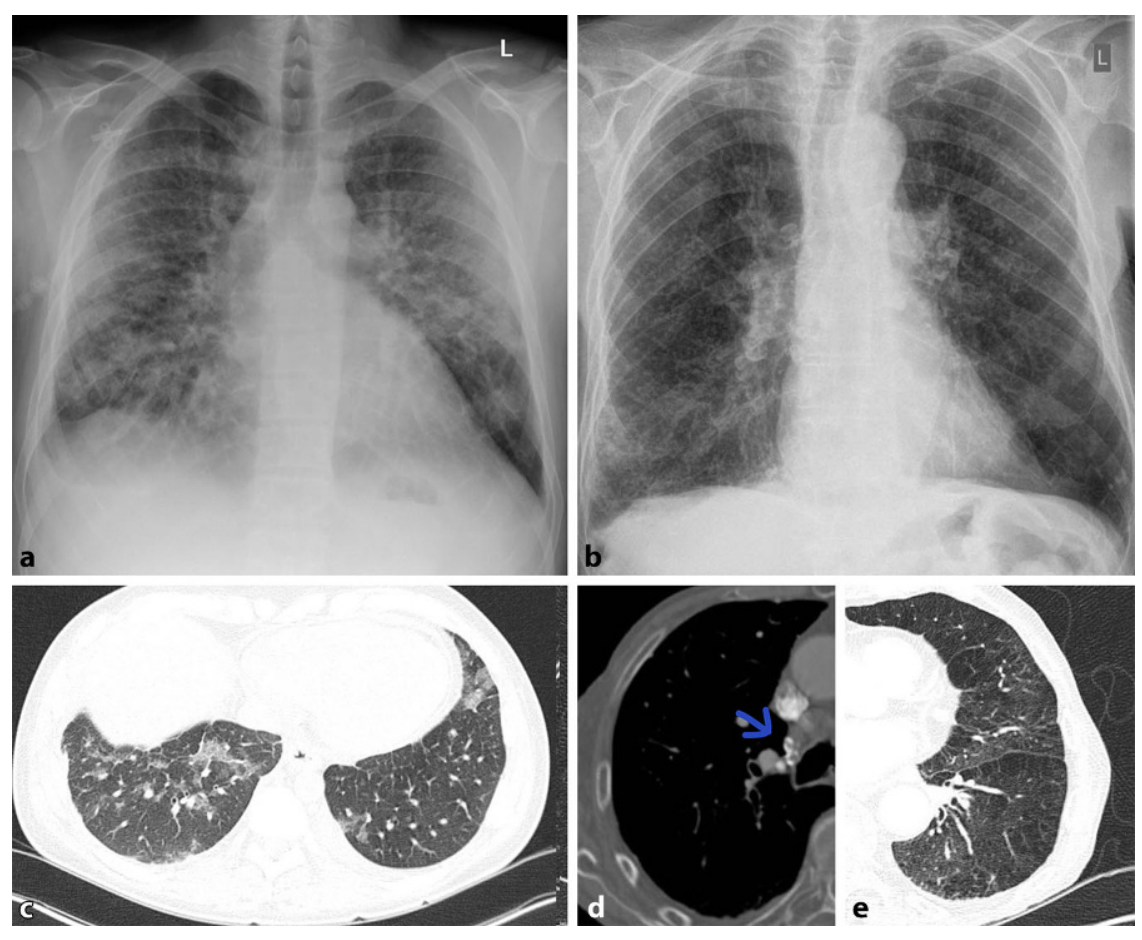

Abb. 1 ॥ Interstitielle Lungenparenchymveränderungen in der konventionellen Röntgenaufnahme im posterior-anterioren (p.-a.) Strahlengang (a) und der dazugehörigen Computertomographie (c) bei atypischer Pneumonie und Silikose (b, e). Eierschalenartige Verkalkungen der Lymphknoten bei Silikose im Weichteilfenster (Pfeil in d)

sache für eine Hospitalisierung dar. Obwohl die Behandlungsmöglichkeiten sich in den letzten Jahrzehnten deutlich verbessert haben, gehört sie mit $14 \%$ Letalität unter den hospitalisierten Patienten zu einer der häufigsten infektionsassoziierten Todesursachen $[6,7]$. Laut statistischem Bundesamt starben im Jahr 2019 deutschlandweit rund 18.500 Menschen aufgrund einer Pneumonie [8].

Die Diagnose der Pneumonie ist prinzipiell eine klinische; die konventionelle Aufnahme dient insbesondere dazu, das Ausmaß der Erkrankung und den Therapieerfolg zu eruieren. Das Erscheinungsbild einer Pneumonie kann jedoch stark variieren. Insbesondere in den Frühphasen können Veränderungen im Röntgenbild fehlen. Studien haben gezeigt, dass $15 \%$ der Pneumonien von Radiologen übersehen werden; Heussel et al. berichten sogar, dass $50 \%$ der Patienten mit einer in der Computertomographie (CT) bestätigten Pneumonie keine charakteristischen Zeichen im Röntgenbild aufweisen [9]. Insbesondere die Art des Verteilungsmusters kann, in sehr beschränktem Ausmaß, jedoch hinweisend auf die Art des Erregers sein und somit dem Kliniker eine Hilfestellung für die weitere Therapie bieten [10].

Allgemein unterscheidet man bei Pneumonien interstitielle von azinären Transparenzminderungen. Interstitielle Pneumonien zeigen in ihrem Erregerspektrum eine große Überlappung mit atypischen Pneumonien, weshalb der Begriff häufig synonym verwendet wird. Interstitielle Veränderungen zeigen ein retikulonoduläres (retikulär $=$ netzartig, nodulär $=$ knotig) Muster (『Abb. 1). Differenzialdiagnostisch ist hier vor allem die Abgrenzung zu interstitiellen Lungenerkrankungen von Bedeutung. Die Sensitivität des konventionellen Bildes wird bei interstitiellen Pneumonien mit nur $40 \%$ berichtet. Bei entsprechendem Risikoprofil, wie Immunsuppression oder Berufsexposition wird daher immer häufiger eine CT zur Primärdiagnostik eingesetzt [11].

Häufige Erreger der interstitiellen Pneumonie sind Viren oder Pilze. Sie grenzen sich in ihrem Erscheinungsbild von bakteriellen Erregern $a b$ und erzeugen milchglasartige Veränderungen, welche zumeist bilateral auftreten. Solche Ground-glassOpazitäten werden aufgrund ihrer gerin- geren Dichte im Röntgenbild jedoch häufig übersehen.

Seit Beginn der COVID-19-Pandemie wächst die Bedeutung der Ground-glassOpazitäten stetig und eine frühe Diagnose ist hier besonders wichtig. Die typischen peripher betonten milchglasartigen Veränderungen sind insbesondere im Anfangsstadium der Erkrankung im konventionellen Bild häufig nur schwer erkennbar; doch eine frühe Diagnose kann für den Patienten entscheidend sein. Aufgrund geringer Übereinstimmung zwischen schwerer Symptomatik und fehlenden Veränderungen in der konventionellen Bildgebung, gilt es, gleichzeitig Differenzialdiagnosen wie eine Lungenembolie schnell und effizient auszuschließen; auch hier ist eine CTUntersuchung in der Primärdiagnostik unverzichtbar ([12]; - Abb. 2).

Azinäre oder alveoläre Verschattungen können sich auf einen Lungenlappen (Lobärpneumonie) oder einzelne Lungensegmente (Bronchopneumonie) beschränken (- Abb. 3). Nicht nur klinisch, sondern auch in ihren bildmorphologischen Zeichen lassen sich diese Pneumonien von der interstitiellen Pneumonie unterscheiden (• Tab. 1).

Konsolidierungen sind alveoläre Füllungsprozesse, bei denen Luft durch Flüssigkeiten wie Mukus ersetzt wird. Die höhere Dichte führt zu einer verstärkten $\mathrm{Ab}$ schwächung der Röntgenstrahlung und dadurch zu einer Verschattung. Dabei können Grenzen von Gefäßen und Bronchialwänden maskiert werden. Konsolidierungen zählen zu den häufigsten Zeichen der Pneumonie. Während sich diese Verschattungen bei einer Lobärpneumonie zunächst auf einen Lappen beschränken, bildet sich bei der Lobärpneumonie häufig das Muster fleckig-konfluierender Konsolidierungen. Hier wird zwar die Grenze der einzelnen Lungensegmente respektiert, jedoch sind häufig mehrere Lungenlappen befallen. Das positive Aerobronchogramm entsteht, wenn Bronchialwege von dicht konsolidierten Lungenabschnitten ummauert sind.

Differenzialdiagnostisch sollte man bei solchen homogenen Verschattungen die segmental oder lobär begrenzt sind, eine Atelektase in Betracht ziehen. Hinweise auf einen Volumenverlust, wie ein 

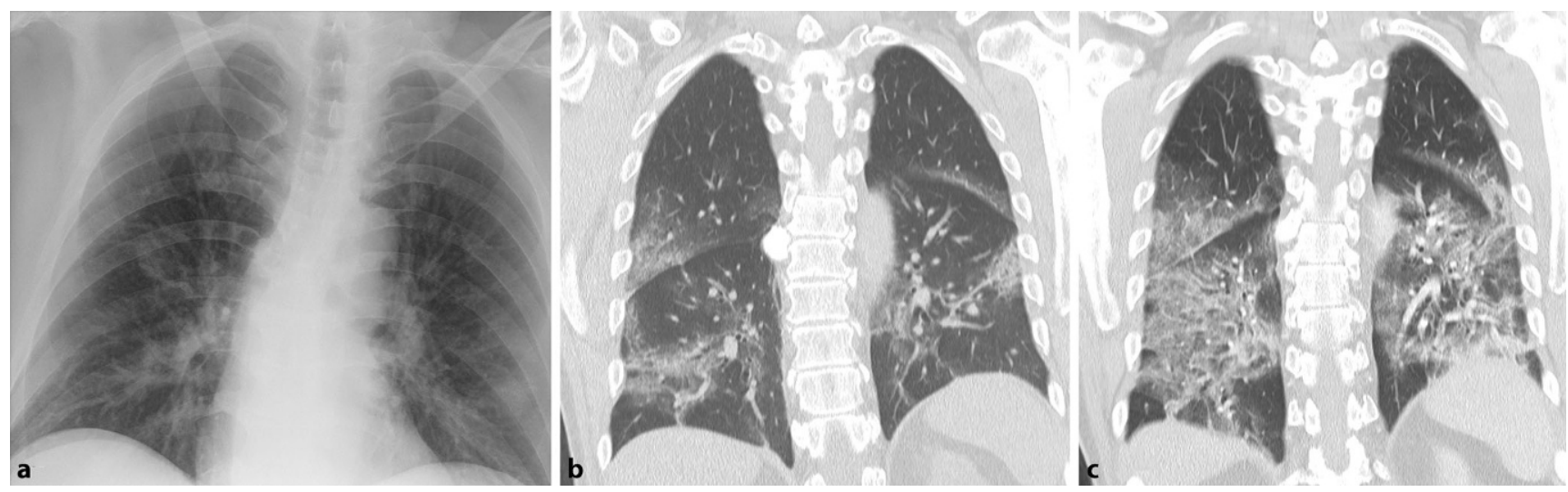

Abb. 2 \ Röntgen-Thorax-Aufnahme im a.-p.-Strahlengang eines 64-jährigen Patienten mit diagnostizierter COVID-19-

Pneumonie mit diskreten Konsolidierungen in beiden Unterlappen (a). Die zugehörige Computertomographie (CT) des Thorax zeigt deutliche Konsolidierungen. Vier Tage später deutlich progredient mit retikulären Veränderungen (b, c
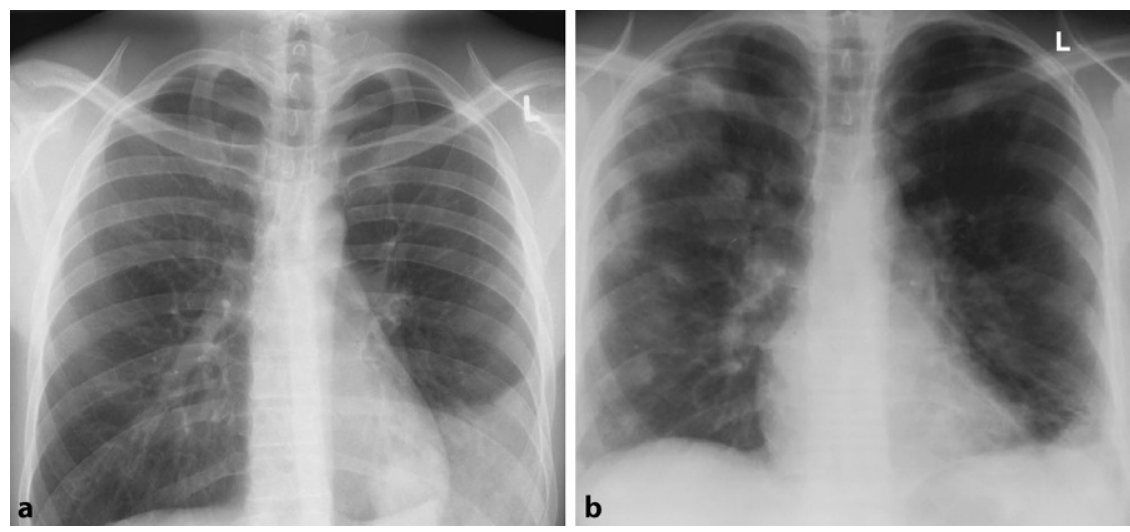

Abb. 3 \& Röntgen-Thorax-Aufnahme im p.-a.-Strahlengang mit Konsolidierung im linken Unterlappen bei Lobärpneumonie (a); fleckig-konfluierende Konsolidierungen mit Befall mehrerer Lungenlappen bei Bronchopneumonie (b)
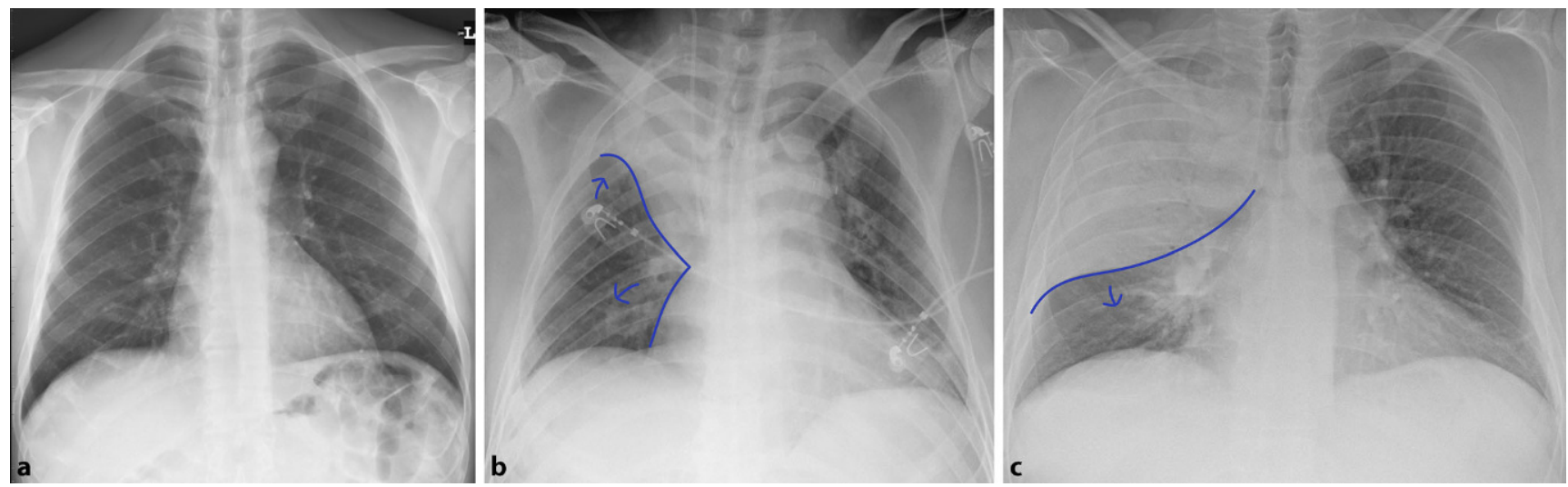

Abb. 4 \ Schematische Darstellung einer Atelektase des rechten Oberlappens mit Verziehung des Hilus und der Fissur (blaue Linie) Richtung Atelektase und geringem Mediastinalshift (Pfeile in b). Infiltrat im linken Oberlappen mit Vorwölbung („,bulging") der Fissur (blaue Linie und Pfeil) und positivem Aerobronchogramm (c); zum Vergleich eine unauffällige RöntgenThorax-Aufnahme im p.-a.-Strahlengang (a) 


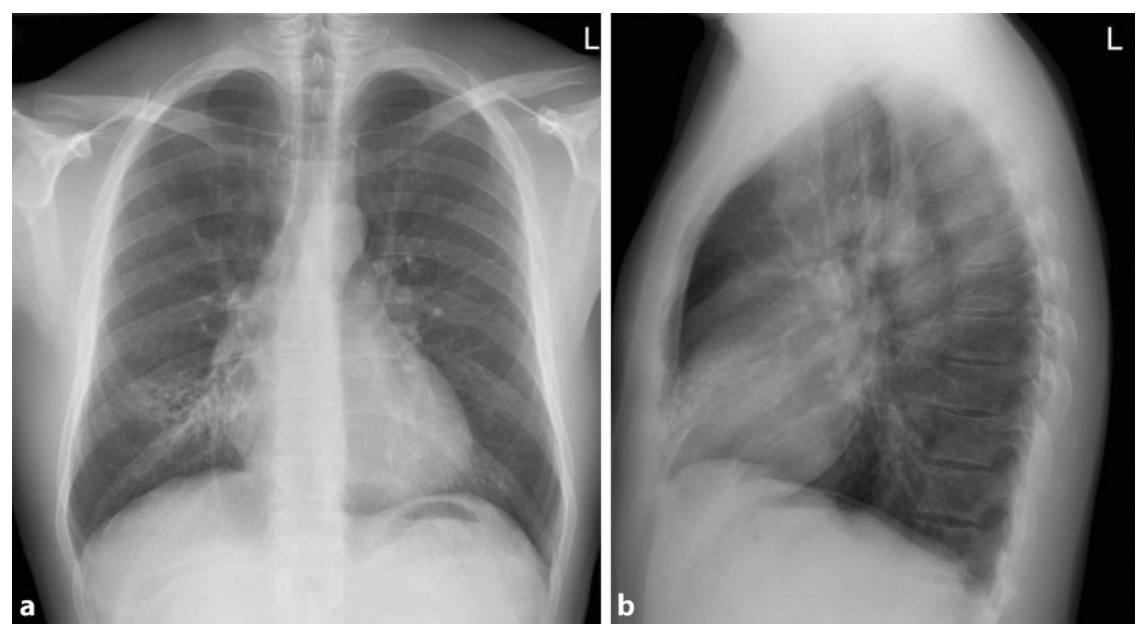

Abb. $5 \Delta$ Der Röntgen-ThoraxAufnahme im p.-a.-Strahlengang zeigt eine Transparenzminderung rechts mit Silhouettenphänomen gegenüber dem rechten Herzschatten (a). Die Projektion im lateralen Strahlengang zeigt deutlich die Lokalisation im Mittellappen (b)
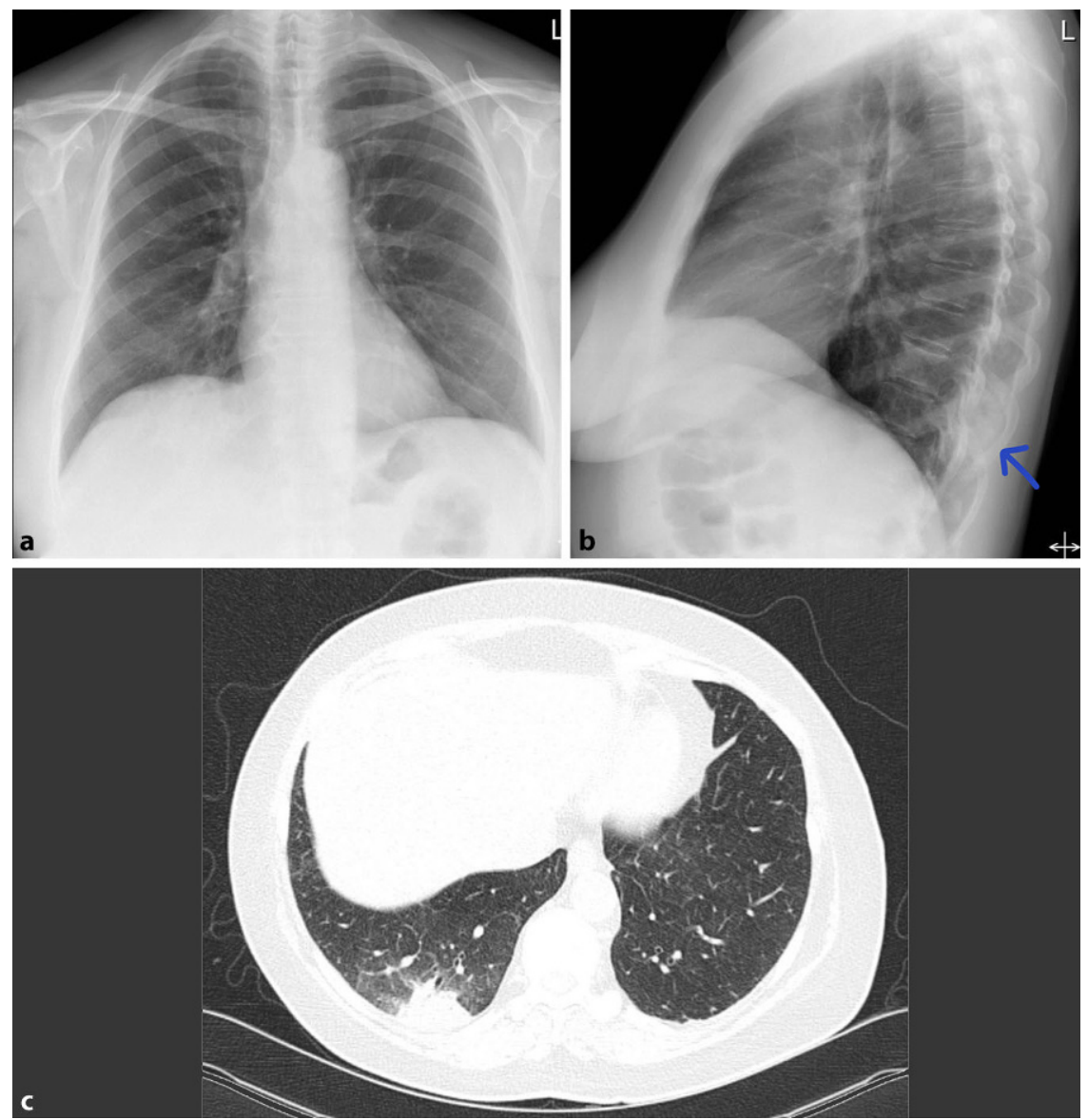

Abb. 6 ム Positives „spine sign“ im lateralen Strahlengang (Pfeil in b) der Röntgen-Thorax-Untersuchung mit Nachweis einer Konsolidierung im rechten Unterlappen in der Computertomographie (c), welche im anterioren Röntgenstrahlengang (a) nicht sichtbar ist
Mediastinalshift oder Verziehungen der interlobären Septen, respektive des Diaphragmas sind für die Unterscheidung hilfreich. Dem gegenüber steht die Volumenzunahme durch ein pneumonisches Infiltrat. Auch hier kann es zu Verschiebungen, in diesem Fall Ausbuchtungen, der Interlobärsepten kommen, dem „bulging fissure sign“ (• Abb. 4). Pathognomonisch ist es für Klebsiella-pneumoniae-Infektionen, hier vor allem im rechten Oberlappen lokalisiert. Die Prävalenz dieses Zeichens ist jedoch insgesamt rückläufig. Nichtinfektiöse Ursachen für eine „bulging fissure" können alveoläre Hämorrhagien, Tumoren und Abszesse sein.

Das Silhouettenzeichen wurde erstmals beschrieben, um Pathologien jeglicher Art anatomisch zuzuordnen. Es beschreibt den Verlust der normalen Grenzflächen zwischen der verdichteten Lunge und Weichteilen und kann durch jeglichen Pathomechanismus ausgelöst werden, bei dem Luft in den Bronchien ersetzt wird [13]. Der Verlust der Grenzflächen, tritt im Röntgenbild immer dann auf, wenn sich die beiden Strukturen gleicher Dichte auf derselben anatomischen Ebene befinden. Ein entzündliches Infiltrat im Mittellappen weist ein Silhouettenphänomen gegenüber dem rechten Herzschatten auf und kann so von einer Konsolidation im rechten Unterlappen differenziert werden. Gleiches gilt für die Lingula und den linken Herzrand (• Abb. 5). Nichtinfektiöse Ursachen für ein positives Silhouettenzeichen können Atelektasen, Aspiration, Pleuraergüsse oder Tumoren sein.

Ein weiteres häufig beschriebenes, jedoch unspezifisches Zeichen ist das „spine sign" im lateralen Strahlengang. In einer normalen Röntgen-Thorax-Untersuchung nimmt die Transparenz der Wirbelkörper zum Zwerchfell hin zu. Eine Transparenzerhöhung spricht bei geringer Sensitivität mit hoher Spezifität für eine Pathologie im Unterlappen ([14]; • Abb. 6).

Als Sonderform des alveolären Musters, ist die miliare Verteilung zu nennen. Sie ist gekennzeichnet durch multiple kleinste pulmonale Noduli $(<3 \mathrm{~mm})$. Eine $\mathrm{mi}-$ liare (Milium, lat.: „Hirsekorn") Verteilung spricht für eine hämorrhagische Streuung des Erregers und ist pathognomonisch für die Tuberkulose, kann jedoch bei immun- 
Tab. 1 Bildmorphologische Charakteristika und häufigste Erreger verschiedener Pneumonien

\begin{tabular}{|l|l|l|}
\hline $\begin{array}{l}\text { Lobär- } \\
\text { pneumonie }\end{array}$ & $\begin{array}{l}\text { Charakteristika } \\
\text { gensegmente oder des ganzen Lappens, be- } \\
\text { grenzt durch Lappenspalten } \\
\text { Positives Aerobronchogramm } \\
\text { Beginnt peripher, setzt sich nach zentral fort } \\
\text { Häufig basale Lungenabschnitte befallen } \\
\text { Häufig Volumenzunahme des betroffenen } \\
\text { Lungenlappens } \\
\text { "Bulging fissure sign" = konvexe Konfiguration } \\
\text { der Lappenspalten bei Klebsielle-Pneumonie }\end{array}$ & $\begin{array}{l}\text { Streptococcus pneumoniae } \\
\text { Legionella pneumophila } \\
\text { Haemophilus influenza }\end{array}$ \\
\hline $\begin{array}{l}\text { Broncho- } \\
\text { pneumonie } \\
=\text { lobuläre } \\
\text { Pneumonie }\end{array}$ & $\begin{array}{l}\text { Unscharf begrenzte Noduli und fleckförmige } \\
\text { Konsolidierungen oder Milchglastrübungen } \\
\text { entlang der Lungensegmente } \\
\text { Häufig mehrere Lungenlappen befallen } \\
\text { Kein Aerobronchogramm } \\
\text { Häufig Atelektasen durch Verlegung der Atem- } \\
\text { wege }\end{array}$ & $\begin{array}{l}\text { Staphylococcus aureus } \\
\text { Streptococcus pneumoniae } \\
\text { E. coli, Haemophilus influenza } \\
\text { Pseudomonas aeroginosa } \\
\text { Viren, Mykoplasmen, Pilze }\end{array}$ \\
\hline $\begin{array}{l}\text { Interstitielle } \\
\text { Pneumonie }\end{array}$ & $\begin{array}{l}\text { Häufig bilateraler, diffuser Befall } \\
\text { Mischbild aus Milchglastrübungen, Konsolidie- } \\
\text { rungen und retikulonodulären Veränderungen }\end{array}$ & $\begin{array}{l}\text { Viren, Mykoplasmen, Pilze } \\
\text { Chlamydien, Rickettsien }\end{array}$ \\
& \multicolumn{2}{|l}{}
\end{tabular}

Tab. 2 Radiologische Muster und ihre Differenzialdiagnosen

\begin{tabular}{|l|l|l|}
\hline Zeichen & Diagnose & Differenzialdiagnose \\
\hline $\begin{array}{l}\text { Konsolidierungen ( } \\
\text { bronchoro- }\end{array}$ & Pneumonie & Atelektase, Tumor, Aspiration \\
\hline Silhouettenzeichen & Pneumonie & Atelektase, Tumor, Erguss \\
\hline „Bulging fissure sign“ & Lobärpneumonie & Abszess, Tumor, Blutung \\
\hline Ground-glass-Opazitäten & Atypische Pneumonie & Lungenödem, Tumor, Vaskulitis \\
\hline „Halo sign" & Aspergillus & $\begin{array}{l}\text { Pseudomonas, HSV, CMV, Granulomatose } \\
\text { mit Polyangiitis }\end{array}$ \\
\hline Luft-Flüssigkeits-Spiegel & Empyem, Abszess & $\begin{array}{l}\text { Tumor(nekrose), Granulomatose mit } \\
\text { Polyangiitis }\end{array}$ \\
\hline "Spine sign“ & Pneumonie & Atelektase, Erguss, Tumor \\
\hline Miliares Muster & Tuberkulose & Varizellen, Metastasen \\
\hline HSV Herpes-simplex-Virus, CMV Zytomegalie-Virus \\
\hline
\end{tabular}

Tab. 3 Empfehlungen für Follow-up-Untersuchungen bei inzidentellen soliden Noduli gemäß Fleischner Society (Version 2017)

\begin{tabular}{|l|l|l|}
\hline & Low-risk & High-risk $^{\mathbf{a}}$ \\
\hline$<6 \mathrm{~mm}$ & Kein Follow-up & Optionale CT in 12 Monaten \\
\hline $6-8 \mathrm{~mm}$ & Solitär: CT in 6-12 und optional in & Solitär: CT in 6-12 Monaten und \\
& 18-24 Monaten & $18-24$ Monaten \\
& Multipel': CT in 3-6 Monaten, optional & Multiple: CT in 3-6 Monaten und \\
& in 18-24 Monaten & $18-24$ Monaten \\
\hline$>8 \mathrm{~mm}$ & Solitär: Intervall CT in 3 Monaten oder & Solitär: Intervall CT in 3 Monaten oder \\
& PET/CT oder Biopsie & PET/CT oder Biopsie \\
& Multiple: CT in 3-6 Monaten, optional & Multiple: CT in 3-6 Monaten und \\
& 18-24 Monaten & 18-24 Monaten \\
\hline
\end{tabular}

CT Computertomographie, PET Positronen-Emissions-Tomographie

${ }^{a}$ Risikofaktoren: Nikotinabusus, Exposition zu Asbest, Radon oder Uran, Lungenkrebs in der Familienanamnese, hohes Alter, weibliches Geschlecht, Ethnie (schwarzafrikanische oder hawaiianische Bevölkerung), spikulierte Berandung, Lokalisation im Oberlappen, Anzahl $<5$, Lungenemphysem oder

-Fibrose

${ }^{b}$ Bei multiplen Noduli zählt der größte Nodulus supprimierten Patienten auch durch andere Erreger ausgelöst werden; exemplarisch zu nennen ist hier die Histoplasmose oder Varizellen-Pneumonie (• Abb. 7). Differenzialdiagnostisch ist an eine diffuse Metastasierung primär bei Melanomen, Schilddrüsen- oder Nierenzellkarzinomen zu denken. Chronische Verschattungen miliaren Musters können selten Zeichen einer Sarkoidose oder einer Pneumokoniose sein und sollten bei entsprechenden Risikofaktoren ebenfalls in Betracht gezogen werden [15].

Pilze grenzen sich durch ein eigenes Erscheinungsbild ab. Sie stellen sich als, teils große, pulmonale Rundherde dar. In der Computertomographie sieht man angrenzend an diesen Rundherd häufig milchglasartige Veränderungen, welche an einen Heiligenschein erinnern; das "halo sign" ist pathognomonisch für eine AspergillusInfektion ([10]; D Abb. 8).

Während o. g. Muster (Ⓣab. 2) häufig unspezifisch sind, kann ein begleitender Pleuraerguss meistens ausreichend und mit hoher Verlässlichkeit beurteilt werden. Charakteristisch ist die homogene Verschattung des kostodiaphragmalen Recessus im lateralen Strahlengang [16].

Differenzialdiagnostisch muss bei gröBeren Verschattungen an einen Hämatothorax oder eine (Teil-)Pneumektomie gedacht werden, was sich mit einem Blick in die Patientenakte schnell evaluieren lässt (- Abb. 9).

Schwieriger ist es bei der Differenzialdiagnose Pleuraempyem. Das typische Muster ist eine linsenförmige Verdickung der Pleura mit Aussparung des Recessus. Jedoch ist eine Beurteilung auch mit einer ergänzenden CT-Untersuchung oft nicht konklusiv möglich.ZusätzlicheZeichen wie eine pleurale Verdickung oder eine vermehrte Kontrastmittelanreicherung treten oft erst spät auf [17].

Komplikationen des Empyems, respektive der fulminanten Pneumonie, ist die bronchopleurale Fistel. Bereits im Röntgenbild würde sich dies durch einen LuftFlüssigkeits-Spiegel im pleuralen Raum darstellen. Differenzialdiagnostisch ist bei Nachweis eines Luft-Flüssigkeits-Spiegels jedoch auch an einen Lungenabszess mit gasbildenden Bakterien zu denken ([15]; - Abb. 10). 

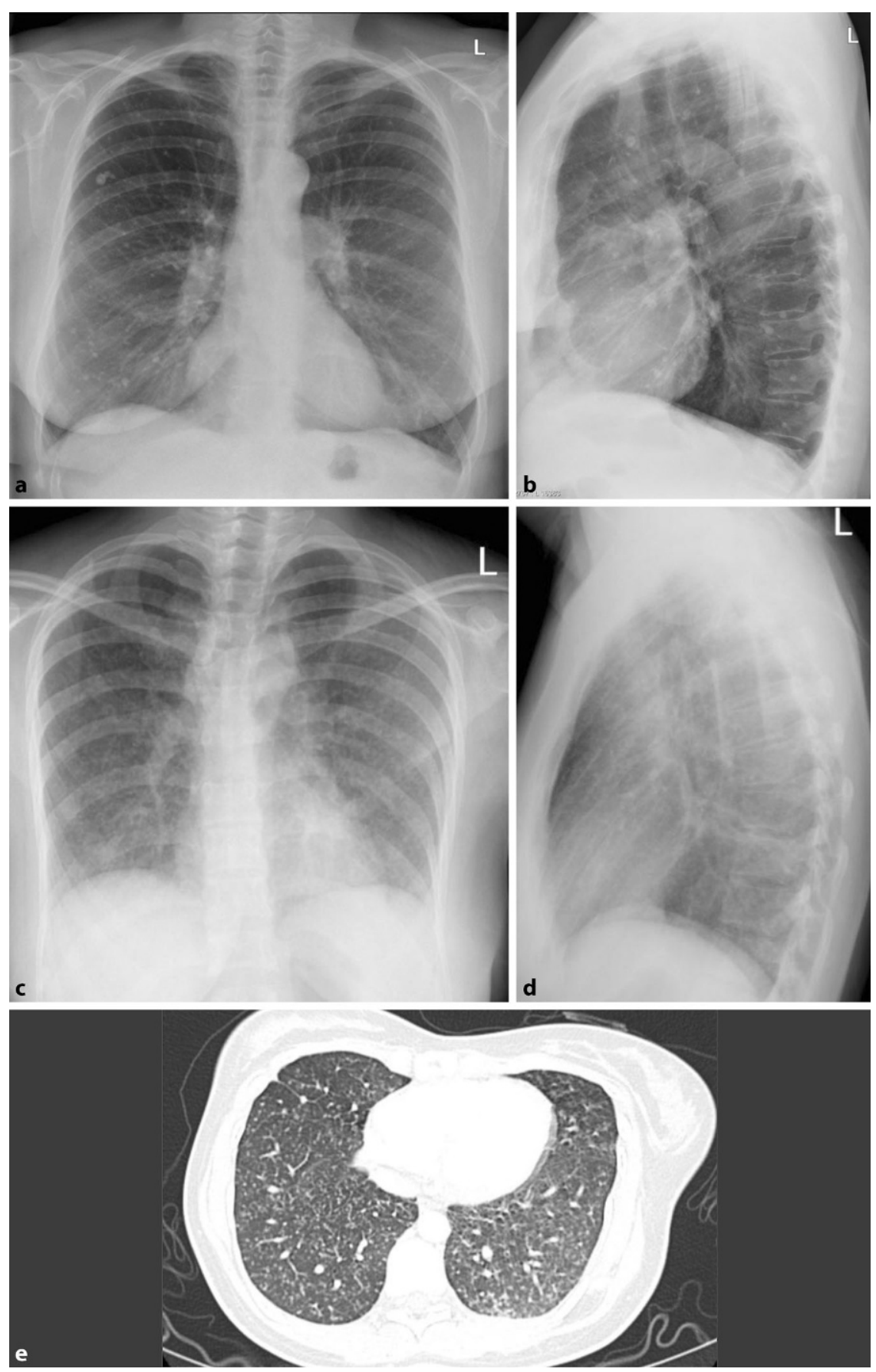

Abb. 7 ॥ Miliares Muster bei Varizellen-Pneumonie $(\mathbf{a}, \mathbf{b})$ und Tuberkulose (c-e) in der Röntgenaufnahme des Thorax in p.-a. und lateralem Strahlengang (a-d) sowie in der zugehörigen Computertomographie (CT) des Thorax (e)

\section{Rolle der Computertomographie - wann ist sie notwendig?}

Fehlende Veränderungen in der Thoraxübersichtsaufnahme allein stellen keine Indikation für eine ergänzende CTUntersuchung dar. Im klinischen Alltag erschweren jedoch zunehmend resistente und atypische Erreger, insbesondere
Pneumonie (PJP) die Röntgenaufnahme des Thorax häufig unauffällig ist, weist die CT-Untersuchung charakteristisch Bild eines "crazy paving" auf. Das typische Mischbild aus bilateralen Ground-glassOpazitäten und Konsolidierungen, meist zentral oder im Oberlappen betont, wird oft begleitet von irregulär verdickten Septen, Kavitäten und Zysten und erinnert mit seinem Muster an ein Mosaik (• Abb. 11; [18]).

Beim immunkompetenten Patienten spielt die Computertomographie bei persistierender Symptomatik insbesondere in der Reevaluation der Therapie eine wichtige Rolle [19]. Bei chronischen und progredienten Konsolidierungen oder „red flags" wie Hämoptoe muss differenzialdiagnostisch auch immer an ein zugrundeliegendes Bronchialkarzinom gedacht werden (• Abb. 12).

\section{Pulmonale Rundherde - nur eine Entzündung?}

Rundliche Transparenzminderungen mit einer $\mathrm{Größe} \mathrm{bis} 3 \mathrm{~cm}$ werden als pulmonale Rundherde bezeichnet. Größere Läsionen, gelten bereits als Raumforderung und somit primär als weiter abklärungsbedürftig. Eine dezidierte Abklärung mittels Computertomographie ist hier notwendig [20].

Die Prävalenz von malignen Läsionen variiert je nach Quelle sehr stark. Nur bei 2-20\% der singulären Rundherde handelt es sich um die Primärmanifestation eines Bronchialkarzinoms. Mehr als die Hälfte der inzidentellen Läsionen sind benigne, wie z.B. Hamartome, Tuberkulome oder Granulome, vaskuläre Läsionen wie Infarkte und Hämatome oder Entzündungen [21]. Morphologische Charakteristika können bei der Risikoeinschätzung pulmonaler Rundherde helfen. Verkalkungsmuster in benignen Läsionen sind jedoch oft unspezifisch, nicht vorhanden oder falschpositiv. So können einige Bronchialkarzinome ebenfalls atypische Verkalkungen aufweisen, welche als Benignitätskriterium fehlinterpretiert werden können [21].

\section{Limitationen des konventionellen Bildes}

Obwohl das konventionelle RöntgenThorax-Bild immer noch zu den Standard- 

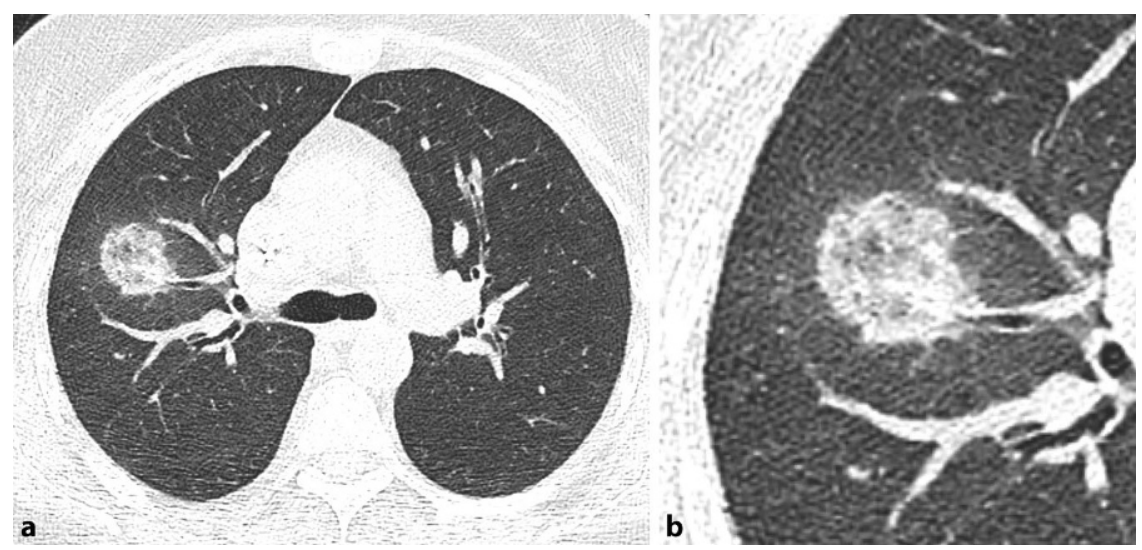

Abb. 8 \ Computertomographie (CT) des Thorax im axialen Schnitt mit pulmonaler Raumforderung im rechten Oberlappen (a). Die vergrößerte Aufnahme zeigt angrenzend einen milchglasartigen Schleier, ein positives „halo sign" bei Aspergillom (b)
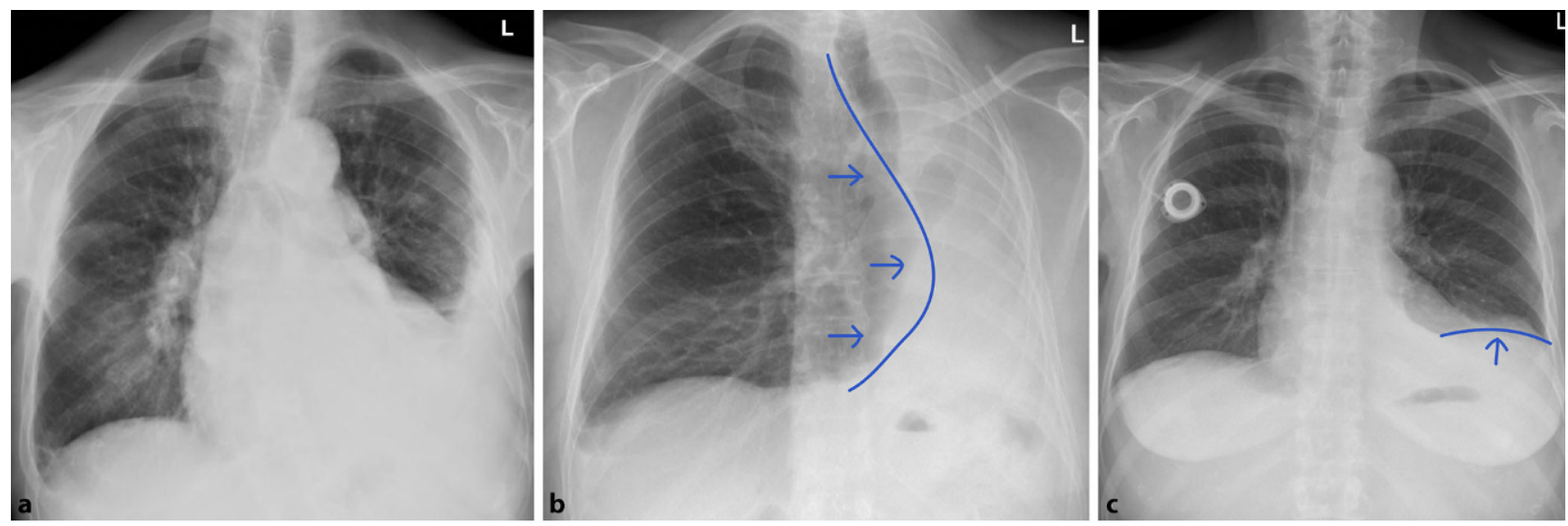

Abb. $9 \Delta$ Röntgen-Thorax-Aufnahme im p.-a.-Strahlengang mit Verschattung des Recessus links bei Pleuraerguss (a). Status nach Pneumektomie mit homogener Transparenzminderung des linken Hemithorax und Mediastinalshift nach links (Pfeile und Linie in b). Teilatelektase des linken Unterlappens mit Zwerchfellhochstand links (Pfeil und Linie in c) bei erhaltenem kostodiaphragmalem Winkel

verfahren in der Primärdiagnostik gehört, ist seine Sensitivität in der Detektion von pulmonalen Rundherden deutlich geringer als die der Computertomographie, daher wird auch die Niedrigdosis-CT und nicht die konventionelle Röntgen-ThoraxUntersuchung in Lungenkarzinomfrüherkennungsprogrammen verwendet [22]. Bei falscher Durchführung ist die RöntgenThorax-Bildgebung aufgrund ihrer Zweidimensionalität deutlich eingeschränkt [5]. Pseudoläsionen können extrapulmonale oder kutane Läsionen, Devices oder Frakturen sein, welche sich als Lungenrundherd darstellen; sie machen $20 \%$ der Lungenopazitäten aus [23]. Standardvorgehen ist deshalb immer die Aufnahme in zwei Ebenen. So lässt sich durch die ergänzende seitliche Thoraxaufnahme schnell zwischen einem Rundherd in der
Mamma oder einer extrathorakalen Installation und einem pulmonalen Nodulus unterscheiden ( $\mathbf{A b b}$. 13). Ist eine Läsion lediglich in einer Ebene darstellbar, sollte man von dem Vorliegen einer Pseudoläsion ausgehen. Ein weiteres Kriterium zur Differenzierung von Pseudoläsionen und pulmonaler Rundherde ist die Berandung. Während Pseudoläsionen keine vollständige Berandung zeigen, sind pulmonale Rundherde vollständig von Lungenparenchym umgeben.

Ob eine Läsion innerhalb des Lungenparenchyms oder doch extrathorakal liegt, lässt sich an dem gemessen Winkel zur Pleura leicht erkennen. Während intrapulmonale Läsionen einen spitzen Winkel zeigen, ist er bei Pseudoläsionen flach (• Abb. 14).
Pleurale Läsionen zählen per Definition ebenfalls zu Pseudoläsionen. Häufig handelt es sich dabei um benigne Plaques. Prädilektionsstellen sind insbesondere die posterolateralen, mediastinalen und diaphragmalen Pleuraabschnitte, die Lungenapeces und kostodiaphragmatischen Winkel bleiben meistens ausgespart. Charakteristische Verkalkungen erleichtern die Diagnose, fehlen jedoch in bis zu $95 \%$ der Pleuraplaques (- Abb. 15). Risikofaktor für die Entstehung pleuraler Plaques ist die Berufsexposition mit Asbest, eine maligne Entartung mit einem meist fulminanten Krankheitsverlauf ist möglich $[24,25]$. 

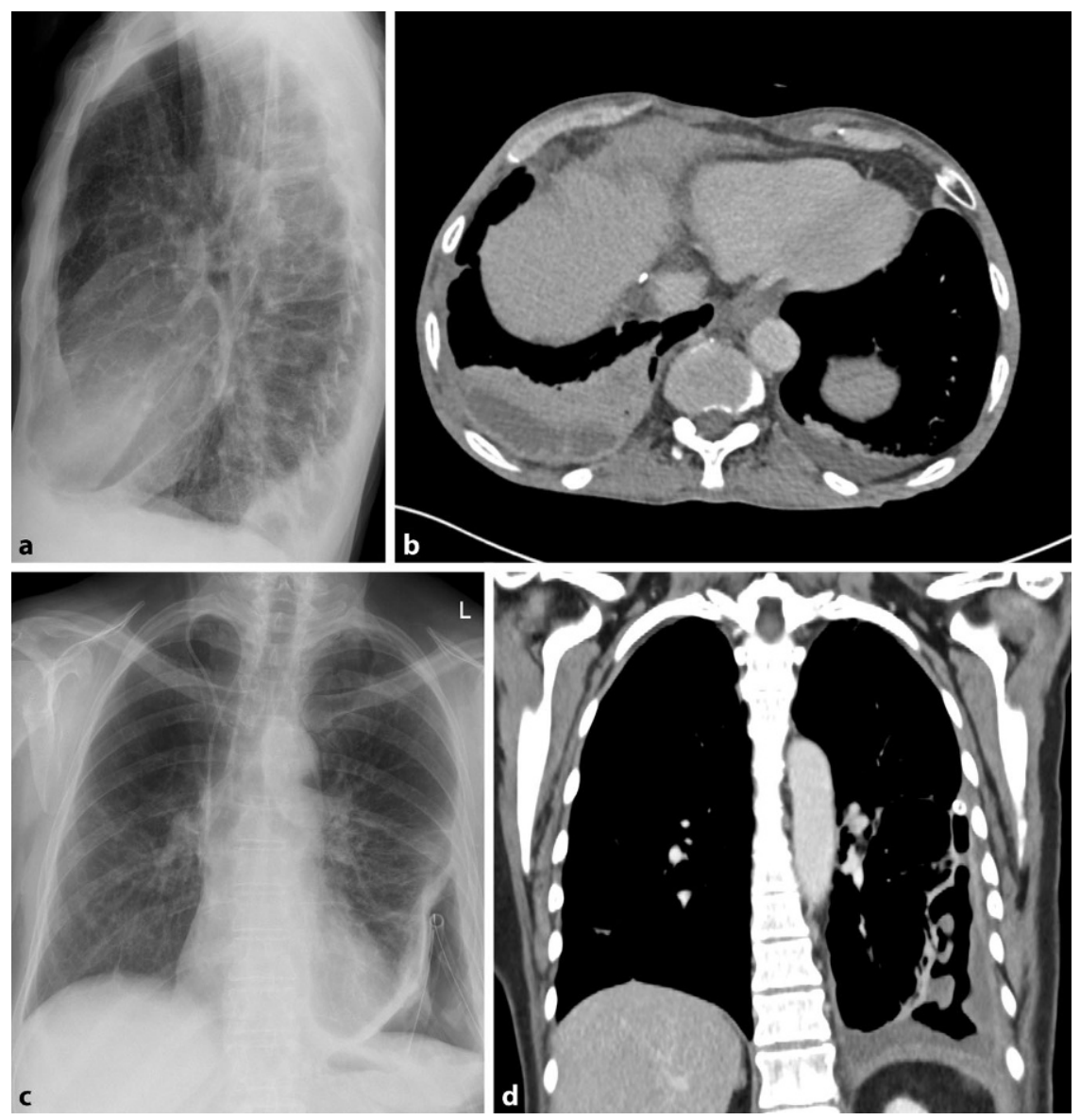

Abb. 10 ム Röntgen-Thorax-Aufnahme im lateralen Strahlengang mit Transparenzminderung rechts dorsobasal (a) mit Nachweis eines Empyems in der zugehörigen Computertomographie des Thorax mit charakteristischer Pleuraverdickung (b). Pleuraempyem mit Luft-Flüssigkeits-Spiegel bei bronchopleuraler Fistel (c, d) in der Röntgenaufnahme des Thorax und der zugehörigen CT-Thorax
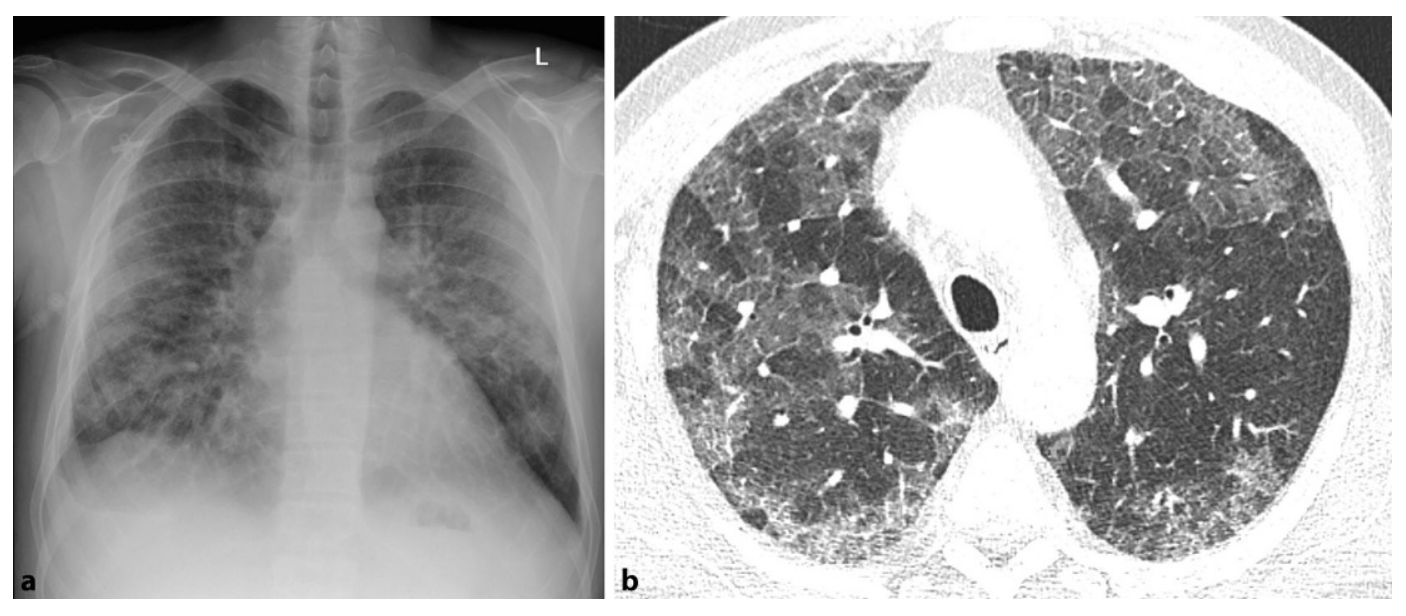

Abb. $11 \triangleleft$ Röntgenbild im p.-a.-Strahlengang (a) mit bilateralen Konsolidierungen. Die zugehörige Computertomographie (b) zeigt ein "crazy paving" beiPneumocystis-jirovecii-Pneumonie (PJP) 

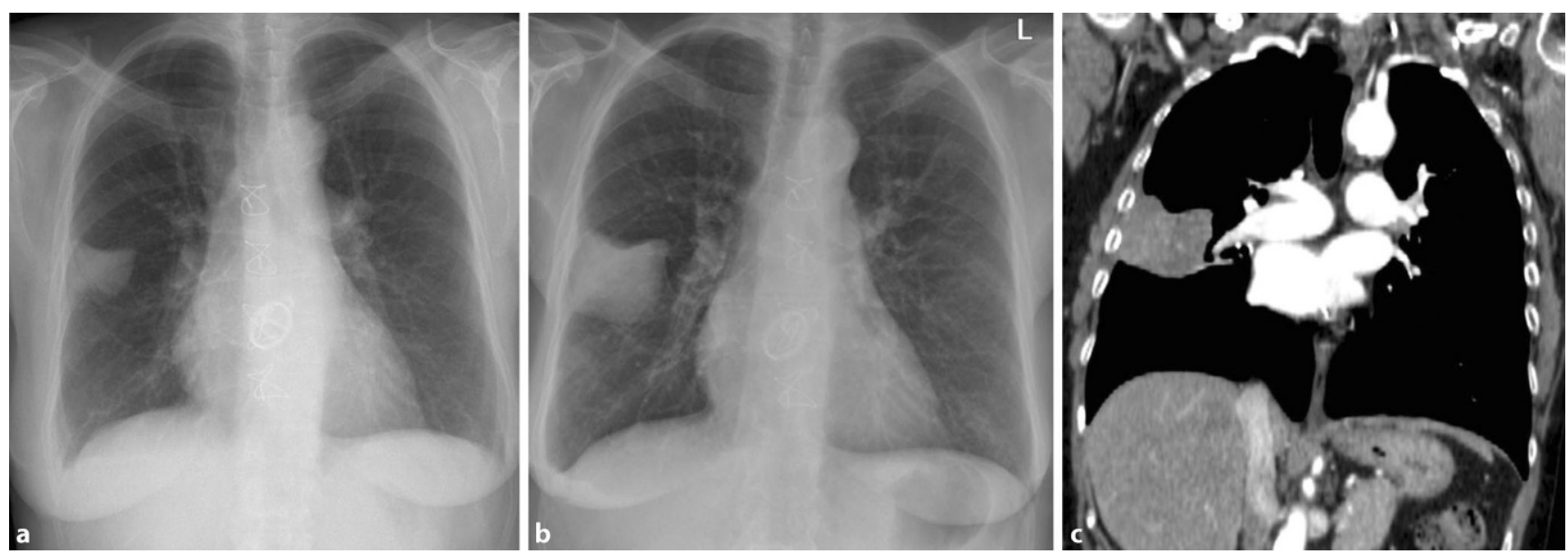

Abb. $12 \Delta$ Röntgen-Thorax-Aufnahme im p.-a.-Strahlengang eines 64-jährigen Patienten mit Husten und Nachweis einer Konsolidierung im rechten Oberlappen (a). Vier Monate nach antibiotischer Therapie Größenprogredienz derselben (b). Die Computertomographie (CT) zeigt ein bronchoalveoläres Karzinom (c)
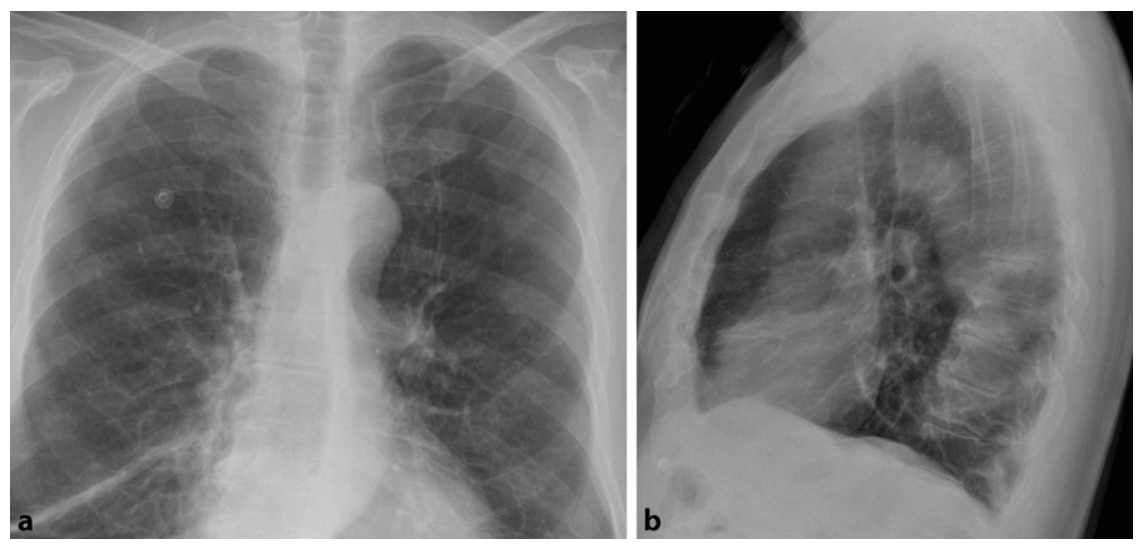

Abb. 13 \ EKG-Elektrode, welche sich im p.-a.-Strahlengang (a) der Röntgen-Thorax-Aufnahme als Rundherd in Projektion auf den rechten Oberlappen präsentiert. Im lateralen Strahlengang (b) nicht darstellbar

\section{Hounsfield-Units: Rolle der Computertomographie}

Durch die Einführung der Computertomographie kam auch die Möglichkeit der Dichtemessung, angegeben in HounsfieldUnits (HU). Diese liefert uns Informationen nicht nur über die Morphologie, sondern auch über die Beschaffenheit einer Läsion. Fett, Flüssigkeit und Verkalkungen zeigen charakteristische HU-Werte und können bei der Klassifizierung von pulmonalen Rundherden hilfreich sein [21]. Einen weiteren Vorteil, den die CT aufbringt, ist die gleichzeitige Beurteilung der umliegenden Strukturen. Wo ein Röntgenbild wenig Auskunft bietet, kann in der CT eine genauere Beurteilung von Weichteilen, Lungenparenchym und ossären Strukturen zum Staging erfolgen. Auch die genaue Lagebeziehung zu angrenzenden Bronchien für die Evaluation einer transbronchialen Biopsie oder CT-gesteuerten Punktion kann beurteilt werden. In fortgeschrittenen Stadien ist vor allem die Infiltration angrenzender Strukturen von prognostischer Bedeutung. Durch die in den letzten Jahren immer besser werdende Technologie wird auch mit einer Low-dose-CT bei deutlicher Dosiseinsparung (Thorax-CT 5,5 mSv vs. Low-dose$\mathrm{CT}<1 \mathrm{mSv}$ vs. konventionelle RöntgenThorax-Aufnahme mit 0,10 mSv; [1]) eine hohe diagnostische Sensitivität erreicht [26]. Dies ermöglicht engmaschige Verlaufskontrollen suspekter Rundherde in der CT und erleichtert dem Radiologen die Beurteilung.

Ein individuelles Assessment bei entsprechendem Risikoprofil zur Evaluation des weiteren Vorgehens ist wichtig und wurde in den letzten Jahren nach mehreren Kriterien optimiert. Die am häufigsten verwendeten Kriterien zur Risikoevaluation von pulmonalen Runderden sind die Fleischner-Kriterien. Berücksichtigt werden hier die Anzahl und das Verteilungsmuster, die Morphologie (Größe, Form und Dichte), aber auch Risikofaktoren, welche ein Bronchialkarzinom begünstigen. Ein häufiger Fehler insbesondere bei jungen Kollegen ist die falsche Messung eines Nodulus (Längsachse $\times$ Kurzachse /2, wobei beide Messungen orthogonal zueinander erfolgen), welche bei entsprechender GröBendynamik Behandlungskonsequenzen nach sich ziehen kann. $\mathbf{0}$ Tab. 3 gibt einen Überblick über die empfohlenen Verlaufsintervalle bei soliden pulmonalen Noduli gemäß Fleischner Society. Bei immunsupprimierten Patienten jedoch sollten die Kriterien nicht angewendet werden, obwohl diese ein erhöhtes Risiko für ein Tumorleiden aufweisen [27].

\section{Die Radiologie im Wandel - Zukunftsaussichten}

Neue Möglichkeiten bietet die in den letzten Jahren immer häufiger angewendete Hybridbildgebung. Hier kann nicht nur die Morphologie eines Rundherdes beurteilt werden, sondern viel wichtiger auch seine biologischen Eigenschaften. So kann es in der CT allein oft schwierig sein, zwischen einer Entzündung, einem Malignom oder postoperativen, respektive posttherapeu- 

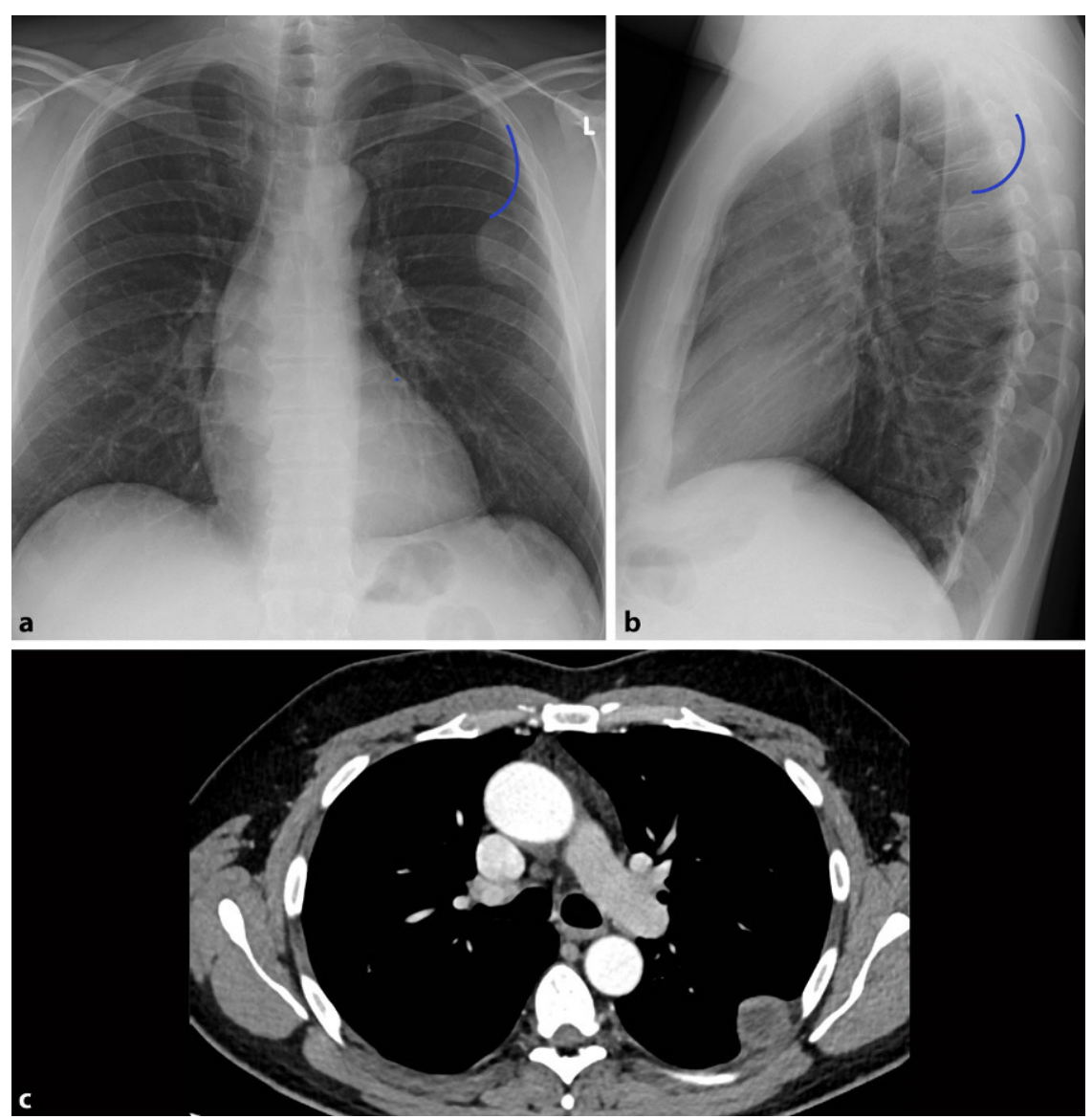

Abb. 14 \ Röntgen-Thorax-Aufnahme mit pleuraständiger Raumforderung im linken Oberlappen mit stumpfem Winkel gegenüber der Pleura im p.-a. und lateralen Strahlengang (Linien in a, b). Die ergänzende Computertomographie (c) weist eine pleurale Raumforderung nach
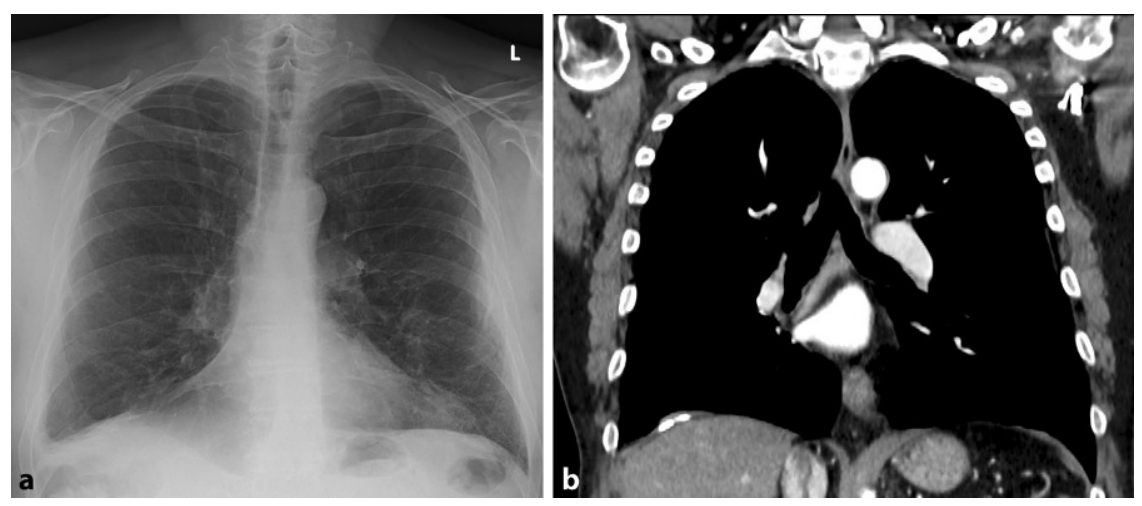

Abb. $15 \Delta$ Röntgen-Thorax-Aufnahme im p.-a.-Strahlengang mit flauer Verkalkung in Projektion auf die rechtsbasale Pleura (a). Nachweis eines verkalkenden Pleuraplaques in der korrelierenden CT-Untersuchung des Thorax (b)

tischen Veränderungen zu unterscheiden. In Deutschland gehört die PositronenEmissions-Tomographie(PET)/CT bereits zur Standarddiagnostik von Bronchialkarzinomen und ist zum Tumorstaging kassenärztlich anerkannt. Insbesondere in der Detektion von Fernmetastasen zeigt haben gezeigt, dass neuronale Netzwerke von Computern mit hoher diagnostischer Genauigkeit pulmonale Noduli erkennen und diese hinsichtlich ihrer Malignitätswahrscheinlichkeit einordnen können [29]. Zum aktuellen Zeitpunkt dienen diese Systeme jedoch eher der Qualitätskontrolle des radiologischen Befundes und sind noch nicht in den klinischen Alltag integriert.

\section{Fazit für die Praxis}

- Das Röntgenbild ist durch seine schnelle Verfügbarkeit weiterhin der Goldstandard in der Primärdiagnostik von Pneumonien.

- Fortschrittliche Techniken erlauben Computertomographie(CT)-Untersuchungen mit vergleichbar geringer Dosis und gewinnen somit an Bedeutung.

- Verteilungsmuster können Hinweise auf die Pathogenese einer Pneumonie liefern, sind häufig jedoch unspezifisch.

- Die Pneumonie ist eine klinische Diagnose. Radiologische Zeichen können im frühen Stadium fehlen.

- Pseudoläsionen sind häufig, können jedoch einfach von pulmonalen Rundherden unterschieden werden.

\section{Korrespondenzadresse}

Prof. Dr. med. Thomas Frauenfelder, MAS Institut für Diagnostische und Interventionelle Radiologie, Universitätsspital Zürich Rämistr. 100, 8091 Zürich, Schweiz thomas.frauenfelder@usz.ch

Funding. Open access funding provided by University of Zurich

\section{Einhaltung ethischer Richtlinien}

Interessenkonflikt. T. Frauenfelder und A. Landsmann geben an, dass kein Interessenkonflikt besteht.

Für diesen Beitrag wurden von den Autoren keine Studien an Menschen oder Tieren durchgeführt. Für die aufgeführten Studien gelten die jeweils dort angegebenen ethischen Richtlinien.

Open Access. Dieser Artikel wird unter der Creative Commons Namensnennung 4.0 International Lizenz veröffentlicht, welche die Nutzung, Vervielfältigung, Bearbeitung, Verbreitung und Wiedergabe in jeglichem Medium und Format erlaubt, sofern Sie den/die ursprünglichen Autor(en) und die Quelle ordnungsgemäß nennen, einen Link zur Creative Commons Lizenz beifügen und angeben, ob Änderungen vorgenommen wurden.

Die in diesem Artikel enthaltenen Bilder und sonstiges Drittmaterial unterliegen ebenfalls der genannten 
Creative Commons Lizenz, sofern sich aus der Abbildungslegende nichts anderes ergibt. Sofern das betreffende Material nicht unter der genannten Creative Commons Lizenz steht und die betreffende Handlung nicht nach gesetzlichen Vorschriften erlaubt ist, ist für die oben aufgeführten Weiterverwendungen des $\mathrm{Ma}$ terials die Einwilligung des jeweiligen Rechteinhabers einzuholen.

Weitere Details zur Lizenz entnehmen Sie bitte der Lizenzinformation auf http://creativecommons.org/ licenses/by/4.0/deed.de.

\section{Literatur}

1. European Union (2014) Medical radiation exposure of the European population. European Comission radiation protection, $\mathrm{Bd} .180$

2. Self WH et al (2013) High discordance of chest $\mathrm{x}$-ray and computed tomography for detection of pulmonary opacities in ED patients: implications for diagnosing pneumonia. Am J Emerg Med 31(2):401-405

3. Makhnevich A et al (2019) The clinical utility of chest radiography for identifying pneumonia: accounting for diagnostic uncertainty in radiology reports. AJR Am J Roentgenol 213(6):1207-1212

4. de Hoop B et al (2010) Screening for lung cancer with digital chest radiography: sensitivity and number of secondary work-up CT examinations. Radiology 255(2):629-637

5. Ebner $L$ et al (2015) Lung nodule detection by microdose $C T$ versus chest radiography (standard and dual-energy subtracted). AJRAm J Roentgenol 204(4):727-735

6. Rider AC, Frazee BW (2018) Community-acquired pneumonia. Emerg Med Clin North Am 36(4):665-683

7. Ewig S (2016) Ambulant erworbene Pneumonie: Epidemiologie. https://www.springermedizin.de/ emedpedia/pneumonie/ambulant-erworbenepneumonie-epidemiologie?epediaDoi $=10.1007$ \%2F978-3-662-45996-6_4.Zugegriffen: 5. Sept. 2021

8. Statista (2021) Todesfälle aufgrund ausgewählter Krankheiten des Atmungssystems in Deutschland von 1998 bis 2019. https:// de.statista.com/statistik/daten/studie/1043878/ umfrage/todesfaelle-aufgrund-ausgewaehlteratemwegserkrankungen-in-deutschland/.Zugegriffen:5. Sept. 2021

9. Heussel CP, Kauczor HU, Ullmann AJ (2004) Pneumonia in neutropenic patients. Eur Radiol 14(2):256-271

10. Hamer $O$ (2013) Erkrankungen der Lunge. In: Hamer O, Zorger N, Feuerbach S, Müller-Wille Retal (Hrsg) Grundkurs Thoraxröntgen. Springer, Berlin, S157-220

11. Dalhoff $\mathrm{K}$ et al (2018) Epidemiology, diagnosis and treatment of adult patients with nosocomial pneumonia-update 2017-S3 guideline of the German Society for Anaesthesiology and Intensive Care Medicine, the German Society for Infectious Diseases, the German Society for Hygiene and Microbiology, the German Respiratory Society and the Paul-Ehrlich-Society for Chemotherapy, the German Radiological Society and the Society for Virology. Pneumologie 72(1):15-63

12. Kwee TC, Kwee RM (2020) Chest CT in COVID-19: what the radiologist needs to know. Radiographics 40(7):1848-1865

\section{Pulmonary nodules and pneumonia. A diagnostic guideline}

Background: Chest X-ray is one of the most frequent examinations in radiology and its interpretation is considered part of the basic knowledge of every radiologist.

Objectives: The purpose of this article is to recognize common signs and patterns of pneumonias and pseudonodules in chest $\mathrm{X}$-rays and to provide a diagnostic guideline for young radiologists.

Materials and methods: Recent studies and data are analyzed and an overview of the most common signs and patterns in chest $\mathrm{X}$-ray is provided.

Results: Knowledge about common signs and patterns in chest $X$-ray is helpful in the diagnosis of pneumonias and can be indicative for the cause of an infection. However, those signs are often unspecific and should, therefore, be set in clinical content.

Computed tomography is becoming increasingly important in the primary diagnosis of pulmonary lesions because of its much higher sensitivity.

Conclusion: Chest X-ray is still the first-line modality in the diagnosis of pneumonia and pulmonary nodules; however, radiologists should be aware of its limitations.

\section{Keywords}

Lung · Infections · Computed tomography · Thoracic radiography · Thorax

13. Felson B, Felson H (1950) Localization of intrathoracic lesions by means of the postero-anterior roentgenogram; the silhouette sign. Radiology 55(3):363-374

14. Medjek M et al (2015) Value of the "spine sign" on lateral chest views. Br J Radiol 88(1050):20140378

15. Walker CM et al (2014) Imaging pulmonary infection: classic signs and patterns. AJR Am J Roentgenol 202(3):479-492

16. Boersma WG et al (2006) Reliability of radiographic findings and the relation to etiologic agents in community-acquired pneumonia. Respir Med 100(5):926-932

17. Reynolds JH et al (2010) Pneumonia in the immunocompetent patient. $\mathrm{Br} \mathrm{J}$ Radiol 83(996):998-1009

18. Kanne JP, Yandow DR, Meyer CA (2012) Pneumocystis jiroveci pneumonia: high-resolution $C T$ findings in patients with and without HIV infection. AJRAm J Roentgenol 198(6):W555-W561

19. Loubet $P$ et al (2020) Community-acquired pneumonia in the emergency department: an algorithm to facilitate diagnosis and guide chest CT scan indication. Clin Microbiol Infect 26(3):382.e1-382.e7

20. Horeweg $\mathrm{N}$ et al (2014) Lung cancer probability in patients with CT-detected pulmonary nodules: a prespecified analysis of data from the NELSON trial of low-dose CT screening. Lancet Oncol 15(12):1332-1341

21. Cruickshank A, Stieler G, Ameer F (2019) Evaluation of the solitary pulmonary nodule. Intern Med J 49(3):306-315

22. Ruparel M et al (2016) Pulmonary nodules and CT screening: the past, present and future. Thorax 71(4):367-375

23. Hansell DM et al (2008) Fleischner Society: glossary of terms for thoracic imaging. Radiology 246(3):697-722

24. Brims FJH et al (2020) Pleural plaques and the risk of lung cancer in asbestos-exposed subjects. Am Respir Crit Care Med 201(1):57-62

25. Kishimoto T et al (1989) Relationship between number of asbestos bodies in autopsy lung and pleural plaques on chest X-ray film. Chest 95(3):549-552
26. Martini K et al (2016) Evaluation of pulmonary nodules and infection on chest $\mathrm{CT}$ with radiation dose equivalent to chest radiography: prospective intra-individual comparison study to standard dose CT. Eur J Radiol 85(2):360-365

27. MacMahon $\mathrm{H}$ et al (2017) Guidelines for management of incidental pulmonary nodules detected on CT images: from the Fleischner Society 2017. Radiology 284(1):228-243

28. Groheux D et al (2016) FDG PET-CT for solitary pulmonary nodule and lung cancer: literature review. Diagn Interv Imaging 97(10):1003-1017

29. Zheng $S$ et al (2021) Deep convolutional neural networks for multiplanar lung nodule detection: improvement in small nodule identification. Med Phys 48(2):733-744 